\title{
Enhancing the Performance of Ultra-Tight Integration of GPS/PL/INS: A Federated Filter Approach
}

\section{Li, J. Wang, S. Babu}

School of Surveying and Spatial Information Systems, University of New South Wales dynamic flight test results of ultra-tight integration based on the federated Kalman filter are presented.

Keywords. GPS, tracking loop, dynamic model, IMU, INS, Kalman filter

\section{Introduction}

The Global Positioning System (GPS), Inertial Navigation System (INS) and Pseudolite (PL) technologies all play very important roles in navigation systems. As an independent navigation system, GPS provides a variety of useful navigation data, e.g. pseudorange, pseudorange-rate, etc. Though the precision is independent of time, the performance will become unreliable when the equipment experiences high dynamics, or when the receiver is exposed to jamming or interference from communication equipment, etc. In comparison to GPS, though INS is autonomous and provides good short-term accuracy, but its usage as a stand-alone navigation system is limited due to the timedependent growth of the inertial sensor biases (Titterton \& Weston, 1997). PLs are ground-based transmitters that can transmit GPS-like signals. They have some advantages in that their positions can be determined precisely, and the Signal-to-Noise Ratios (SNR) are relatively high. The integration of GPS, INS and PL is increasingly important, because their combined performance, in principle, overcomes the shortcomings of the individual sensor systems.

Initially, the GPS and INS were integrated in "loose" mode, where the navigation solutions from the individual systems are combined together by an optimal integrating filter. In this system-level integration, GPS and INS systems were treated independently. Though easy to implement, the navigation solution can be improved. As a 
result, the integration evolved into the so-called tightlycoupled mode, where the raw measurements from GPS, e.g. pseudorange, pseudorange-rate, are combined with INS measurements or positions (Sennott, 1997). The system performance is remarkablely improved.

In severe environments, such as under high dynamics, and/or intentional or unintentional RF interference, the performance of the loose and tightly-integrated system will be degraded because the GPS solutions become increasingly unreliable. For a more robust navigation solution, the integration level moves into both the individual systems, especially into the GPS receiver itself. That is, the ultra-tight integration navigation filter combines either the I and Q measurements from the GPS tracking loop or the pseudorange measurements with the INS navigation parameters to produce the optimal Doppler estimate. This removes the dynamic stress of the code and phase tracking loops, thus keeping them in stable tracking mode during high dynamics (Alban, 2003; Beser, 2002; Poh, 2002). This tracking loop level integration is more complicated than the other two as it requires good knowledge of GPS receiver's tracking loop architecture. Differing from the conventional GPS receiver used in the other two configurations, in which the tracking loop is closed and the local reference signal is the only feedback of the loop filter, the ultra-tightly integrated system's feedback signal is derived from both the loop filter and the integrated navigation filter. This improves the accuracy of GPS measurements by maintaining a narrow tracking loop bandwidth without degrading its dynamic tracking capability.

In an ultra-tightly integrated system, usually the updating frequency of the GPS tracking loop is $1 \mathrm{kHz}$. However, due to large-sized matrix computations and hardware limitations, the integrated Kalman filter output rate is between $1-10 \mathrm{~Hz}$. Accurate estimates need a complicated filter structure, which typically means more computing time. For real-time applications, not only the precision of estimates, but also the computing time should be taken into account. Hence there must be a compromise in the system design.

This paper proposes a federated Kalman filter structure to implement the ultra-tight integration. The computation by the integrated filter is distributed into different parts, so that the main filter integrates the GPS measurements and INS data while the other filter, i.e. pre-filtering filter, is specifically designed to compensate for the IMU errors. These two Kalman filters work in parallel, so that the computing time is kept to a minimum. When using a Kalman filter to estimate and compensate for the IMU errors, a pivotal technique is to determine the dynamic model of the IMU sensors, which is used to derive the state transition matrix for implementing the Kalman filter. This paper will discuss the dynamic modelling of an IMU employing a novel modelling method based on the Walsh function and its transform. The precision of the dynamic model of the IMU directly influences the accuracy of the INS data, and eventually the quality of the aiding Doppler. In this paper, a dynamic modelling method will be discussed and simulation experiments will be carried out to demonstrate the proposed dynamic modelling method. Several test scenarios have been used to investigate the ultra-tight receiver based on the federated Kalman filter structure, compared to a standalone receiver. The results show that the ultra-tight integration produces a more robust and accurate solution under high dynamics and low SNR environments.

\section{Kalman Filter Structure for The Ultra-Tight Integration}

The purpose of the ultra-tight integration is to keep the GPS receiver stable for high dynamic applications, with the integration of inertial measurements. As the dynamics are removed from the tracking loop, the loop bandwidth will be reduced to the minimum, depending on the accuracy of the aiding measurements derived from the inertial sensors and the stability of the receiver clock. As a result, in this case, even in high dynamics and low SNR applications the tracking loop could remain in a narrow PLL, which means precise measurements and therefore an accurate and robust position solution.

In general, in either the ultra-tight integration or the stand-alone GPS receiver operation, the loop filter measures the errors between the incoming and reference signals and feeds them into the Numeric Control Oscillator (NCO) to align the phase of the local reference signal so that it's frequency and phase are identical to those of the incoming signal. Usually this process will take 1 millisecond, i.e. one $\mathrm{C} / \mathrm{A}$ code period. The update rate of the loop is $1 \mathrm{kHz}$. In the ultra-tight integration, the aiding Doppler from the integrated filter should be provided at the same rate.

The integrated filter which outputs the aiding Doppler is implemented by Kalman filter techniques. Because there are intensive computations the computing time of the Kalman filter is longer than 1 millisecond; resulting in a data update rate of $1-10 \mathrm{~Hz}$. To synchronise the tracking loop and the aiding Doppler, the output rate of the Kalman filter must be increased by simplifying its structure (but which would otherwise result in a degradation of the quality of the aiding Doppler and other estimates). One method to solve this problem is to interpolate the lower aiding Doppler rate to the required rate, i.e. $1 \mathrm{kHz}$, with a multi-rate signal processing algorithm (Babu and Wang, 2004). For improved estimates, the Kalman filter should be carefully designed. However, the long computation time for accurate results will reduce the output rate and result in degradation of the performance of the interpolation. 
To solve this paradox there must be a compromise between the computing time and the estimating accuracy of the Kalman filter. The federated Kalman filter structure is investigated to implement the ultra-tight integration, whereby the computation of the integrated filter is divided into different filters. Figure 1 depicts the system structure of ultra-tight integration based on a federated Kalman filter. The main filter integrates the GPS measurements and the INS data, while the other one, i.e. the pre-filtering Kalman filter, will operate in parallel with the main filter to estimate and compensate for the IMU errors. This structure succeeds in distributing the computational burden into different filters, all operating at the same time.

The Kalman filter is used to generate optimal estimates of a dynamic system. The key to implementing the prefiltering Kalman filter is to determine its state transition matrix, which could be directly deduced from the dynamic model of the estimated system. For describing the characteristics of the dynamic system, we can use the dynamic model or the state transition matrix.

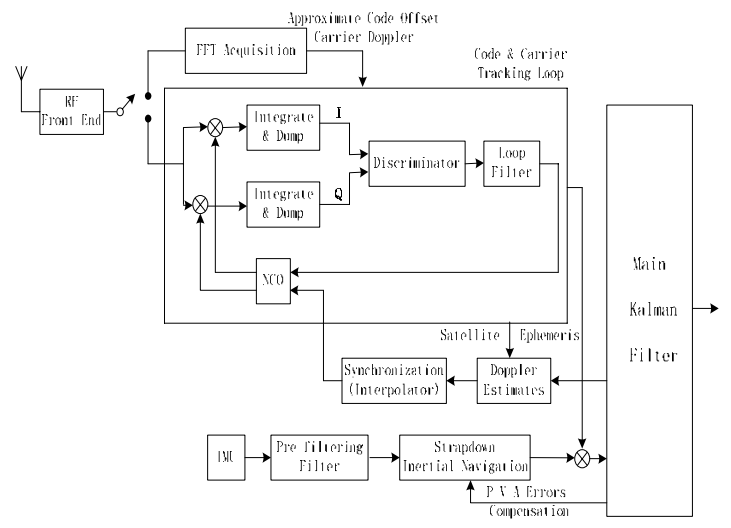

Fig. 1 Configuration of the federated Kalman filter for the ultra tight integration

To implement the pre-filtering Kalman filter requires a knowledge of the IMU dynamic model. Usually there are time-domain and frequency-domain based methods to establish this dynamic model. However these two methods are inefficient because they are computationally intensive, complex and it is easy to introduce errors in the multi-modelling steps ( $\mathrm{Li}, 2004)$. In this paper the IMU dynamic model is derived by a new dynamic modelling method based on the Walsh function and its transform. The advantages of this modelling method are that the integration can be replaced by matrix multiplication, and the parameters of the dynamic model can be directly derived from the matrix operation. Thus the errors can be reduced to obtain a more accurate model of the dynamic system.

\section{Implementation of The Federated Kalman Filter}

\subsection{Implementation of The Pre-filtering Kalman Filter}

The effective way to eliminate the errors of the IMU is the complementary Kalman filter technique. Based on the Kalman filter theory, precise estimates can only be derived from the accurate dynamic model.

Usually the characteristic of the dynamic system is described by means of a transfer function, which is the representation of the differential equation in the sdomain. The IMU dynamic model can be represented by $1^{\text {st }}$ or $2^{\text {nd }}$ order transfer functions (Li and Sun, 2004). The outputs of the IMU should be estimated in real-time, which usually has a high output rate. In this case, the $1^{\text {st }}$ order IMU models are used to simplify the structure of the pre-filtering Kalman filter and, as a result, this increases the output rate. The transfer function of IMU can be given as:

$\Phi(s)=\frac{K}{T s+1}$

where $K$ is the system gain and $T$ is the time constant of system. These two parameters have an impact on the system dynamic performance. The determination of the IMU dynamic model becomes a problem of parameter identification in equation (1). Using the Walsh function dynamic modelling method, firstly the calibrated data are collected from the IMU. Secondly, $W_{m}, p$ and $\omega^{T}$ are generated. Then the Walsh transform of input/output data are carried out. And finally the coefficients $K$ and $T$ are extracted by matrix operations.

Once $K$ and $T$ have been determined and the noise $w$ has been introduced, the transfer function equation (1) can be transformed into a state-space description:

$\left\{\begin{array}{c}\dot{x}=-\frac{1}{T} x+w \\ y=x+v\end{array}\right.$

where $T$ is the time constant, and $w$ is the system noise. Discretising the continuous state-space equation (2) (Zheng, 2000):

$$
\left\{\begin{array} { c } 
{ X ( k + 1 ) = G X ( K ) + H W } \\
{ Y ( k ) = X ( k ) + V }
\end{array} \left\{\begin{array}{c}
G=e^{-\frac{T_{s}}{T}} \\
H=\int_{0}^{T_{s}} e^{-\frac{1}{T} t} d t
\end{array}\right.\right.
$$

where Ts is the sampling period, $\mathrm{T}$ is the time constant of system, $G$ is the system state transition matrix, $H$ is the system noise transition matrix, the process and measurement model of the Kalman filter can be derived using the dynamic model of the IMU: 
$\left\{\begin{array}{c}\Phi_{I M U}(k+1)=G \Phi_{I M U}(k)+H w(k) \\ Y_{I M U}(k)=\Phi_{I M U}(k)+v(k)\end{array}\right.$

where $\Phi_{I M U}=\left[\varphi_{\text {gyrol }}, \varphi_{\text {gyro } 2}, \varphi_{\text {gyro } 2}, \varphi_{\text {accl }}, \varphi_{\text {acc } 2}, \varphi_{a c c 3}\right]^{T}$, is the state vector of the Kalman filter. Here $\varphi_{\text {gyrol }}, \varphi_{\text {gyro2 }}, \varphi_{\text {gyro2 } 2}, \varphi_{\text {accl }}, \varphi_{\text {acc } 2}, \varphi_{\text {acc } 3}$ are the estimated outputs of the IMU, and $w(k), v(k)$ are zeromean white noise. $Y_{I M U}=\left[y_{\text {gyro 1 }}, y_{\text {gyro 2 }}, y_{\text {gyro 3 }}, y_{\text {acc 1 }}, y_{\text {acc } 2}, y_{\text {acc } 3}\right]^{T}$ and $y_{\text {gyro1 }}, y_{\text {gyro } 2}, y_{\text {gyro } 3}, y_{a c c 1}, y_{a c c 2}, y_{a c c 3}$ are the original outputs of the IMU used as the measurements for the prefiltering Kalman filter.

When the original outputs of the IMU are used as the measurements of the pre-filtering Kalman filter, the estimated outputs are the optimal estimation of the raw outputs of the IMU, i.e. a pre-filtering Kalman filter can produce the optimal estimates of the IMU outputs. Hence, these calibrated IMU outputs derived from the prefiltering Kalman filter can be used in the inertial navigation algorithm to generate the navigation solutions for position, velocity and attitude.

\subsection{The Simplified Structure of the Main Kalman Filter}

The main advantage of the federated Kalman filter is that it can distribute the computational tasks of a centralised Kalman filter into two parallell Kalman filters, i.e. the pre-filtering and the main Kalman filter. The structure of main Kalman filter can be simplified at the same time without degrading the quality of the estimates. The IMU errors, i.e. the gyro and accelerometer random drifts, are estimated and compensated for in the pre-filter.

There are several different options for implementing the ultra-tight integration Kalman filter. In one of them, the GPS-measured pseudorange is used as the measurement for the Kalman filter. Based on the optimal estimates of receiver velocity, the aiding Doppler can be obtained to feed back the tracking loop (Alban, 2003). In this paper, this structure is adopted to implement the ultra-tight integration. Usually the state vector in this type of ultratight Kalman filter structure comprises 16 variables:

$$
\begin{aligned}
x(t)=\left[\begin{array}{llllllllll}
\delta \varphi & \delta \lambda & \delta h & \delta v_{e} & \delta v_{n} & \delta v_{z} & \alpha & \beta & \gamma \\
& \delta f_{i b x}^{b} & \delta f_{i b y}^{b} & \delta f_{i b z}^{b} & \delta \omega_{i b x}^{b} & \delta \omega_{i b y}^{b} & \delta \omega_{i b z}^{b} & \delta t_{b}
\end{array}\right]
\end{aligned}
$$

where $\delta \varphi, \delta \lambda, \delta h$ are the position errors, i.e. latitude, longitude and height errors. $\delta v_{e}, \delta v_{n}, \delta v_{z}$ are the velocity errors, i.e. east, north and up velocity errors in the local level coordinate system, respectively. $\alpha, \beta, \gamma$ are the attitude errors, i.e. pitch, roll and heading angle errors in the body coordinate system. $\delta f_{i b x}^{b}, \delta f_{i b y}^{b}, \delta f_{i b z}^{b}$ are the 3 accelerometer random drifts, $\delta \omega_{i b x}^{b}, \delta \omega_{i b y}^{b}, \delta \omega_{i b z}^{b}$ are the 3 gyro random drifts, and $\delta t_{b}$ is the GPS receiver clock bias.

The measurement used in the Kalman filter is the difference between the INS-derived pseudorange and the GPS-measured pseudorange; therefore it is a function of receiver position errors and GPS receiver clock bias errors:

$\delta \rho=e_{1} \delta \varphi+e_{2} \delta \lambda+e_{3} \delta h+\delta t_{b}+V$

where $e_{1}, e_{2}, e_{3}$ are the elements of the unit vector between satellite and receiver. $V$ is the measurement noise. $\delta \rho$ is the pseudorange difference, which is derived from $\rho_{I N S}-\rho_{G P S}$, here $\rho_{I N S}$ is the INS-derived pseudorange measurement which contains the receiver position errors, and $\rho_{G P S}$ is the GPS-measured pseudorange which contains the receiver clock bias errors.

Once we obtain the optimal estimates of receiver velocity, the aiding Doppler can be calculated:

$f_{\text {doppler }}=f_{t x} \frac{\left(1-v_{v e l} \vec{a}\right)}{c}$

where $f_{t x}$ is the GPS L1 frequency, $V_{v e l}$ is the relative velocity between satellite and receiver, $\vec{a}$ is the line-ofsight unit vector between satellite and receiver, and $c$ is the speed of light. For a detailed discussion of how to derive the aiding Doppler from the estimated velocity readers are referred to Babu and Wang (2004) and Kaplan (1996).

In the federated Kalman filter structure, as the prefiltering Kalman filter compensates for the IMU errors, there is no need for the main Kalman filter to estimate the IMU random errors, therefore the number of state variables can be reduced to 10 (removing the $6 \mathrm{IMU}$ drift state variables), and the structure of the Kalman filter (i.e. the state transition matrix) can be simplified. If the number of state variables is 16 or 10 , the number of elements in the state transition matrix will be $16^{2}=256$ or $10^{2}=100$ respectively. Hence because the value of every element should be calculated by the software, reducing the number of state variables can significantly reduce the computational burden of the Kalman filter. Furthermore, reducing the state variables can simplify the structure of the Kalman filter by neglecting the correlations between the IMU errors and the other variables. Because the attitude and velocity errors are correlated with the IMU random drift errors, for example, the attitude pitch angle and the east velocity error equations are given as:

$\dot{\alpha}=-\frac{\delta v_{n}}{R}+\left(\omega_{i e} \sin \varphi+\frac{v_{e}}{R} \operatorname{tg} \varphi\right) \beta-\left(\omega_{i e} \cos \varphi+\frac{v_{e}}{R}\right) \gamma-\delta \omega_{i b x}^{b}$ 


$$
\begin{aligned}
\delta \dot{v}_{n}= & f_{z} \alpha-f_{x} \gamma+\delta i_{i b y}^{b}-2\left(\omega_{i e} \sin \varphi+\frac{v_{e}}{R} \operatorname{tg} \delta\right) \delta v_{e} \\
& -\left(2 \omega_{i e} \cos \varphi+\frac{v_{e}}{R} \sec ^{2} \varphi\right) v_{e} \delta \varphi-\frac{v_{n}}{R} \delta v_{u}-\frac{v_{u}}{R} \delta v_{n}
\end{aligned}
$$

where $R=R_{e}+h, R_{e}$ and $h$ is the earth radius and height respectively, and $\omega_{i e}$ is the angle rate of earth rotation. $f_{x}, f_{z}$ are the accelerometer measurements in the body coordinate system.

In the federated Kalman filter structure, the pre-filtering Kalman filter estimates and compensates for the IMU random drifts, thus the main Kalman filter doesn't need to estimate them. As described above, the correlations of the IMU random drift errors with the other state variables can be neglected. The attitude and velocity error equation could be simplified:

$$
\begin{aligned}
\dot{\alpha}= & -\frac{\delta v_{n}}{R}+\left(\omega_{i e} \sin \varphi+\frac{v_{e}}{R} \operatorname{tg} \varphi\right) \beta-\left(\omega_{i e} \cos \varphi+\frac{v_{e}}{R}\right) \gamma \\
\delta \dot{v}_{n}= & f_{z} \alpha-f_{x} \gamma-2\left(\omega_{i e} \sin \varphi+\frac{v_{e}}{R} \operatorname{tg} \delta\right) \delta v_{e} \\
& -\left(2 \omega_{i e} \cos \varphi+\frac{v_{e}}{R} \sec ^{2} \varphi\right) v_{e} \delta \varphi-\frac{v_{n}}{R} \delta v_{u}-\frac{v_{u}}{R} \delta v_{n}
\end{aligned}
$$

Hence the state transition matrix can be simplified. Here we still use the pseudorange differences as the measurements for the Kalman filter. Hence the measurement model is the same as that in the centralised Kalman filter, which is described by equation (6).

\section{Experimental Results}

\subsection{IMU Dynamic Modelling Results}

Given the 2nd order dynamic system:

$$
G(s)=\frac{b_{1} s+b_{2}}{s^{2}+a_{1} s+a_{2}}=\frac{1}{s^{2}+0.53 s+1}
$$

the Walsh function modelling results are shown in Table 1. Using these modelling results the dynamic models are constructed, as shown in Figure 2. The dashed lines represent the outputs of these models and the solid lines represent the outputs of the model in equation (10) which were applied to the same unit step input signal.

Table 1. Modeling results of method using different numbers of Walsh function

\begin{tabular}{c|c|c|c|c}
\hline $\begin{array}{c}\text { Model } \\
\text { Parameters }\end{array}$ & $a_{1}$ & $a_{2}$ & $b_{1}$ & $b_{2}$ \\
\hline $\begin{array}{c}8 \text { Walsh } \\
\text { function }\end{array}$ & 0.53 & 1 & 0 & 1 \\
\hline $\begin{array}{c}32 \text { Walsh } \\
\text { function }\end{array}$ & 0.54293 & 1.0162 & -0.16551 & 1.0163 \\
\hline $\begin{array}{c}\text { 128 Walsh } \\
\text { function }\end{array}$ & 0.53035 & 1.0009 & $\begin{array}{c}- \\
0.0054747\end{array}$ & 1.001 \\
\hline $\begin{array}{c}256 \text { Walsh } \\
\text { function }\end{array}$ & 0.53018 & 1.0002 & -0.020797 & 1.0002 \\
\hline
\end{tabular}

The results show that the accuracy of the estimated model parameters depends on the number of Walsh functions used for the modelling. For the simulation test, 128 Walsh functions produce good modelling results.

\subsection{Pre-filtering Kalman Filter Testing Results}

First, the flight trajectory with known dynamics, with characteristics shown in Table 2, was generated.
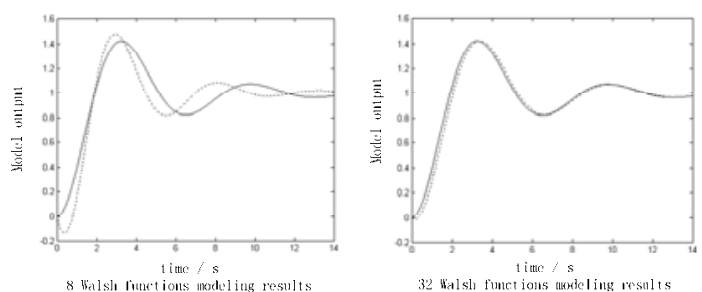

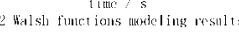
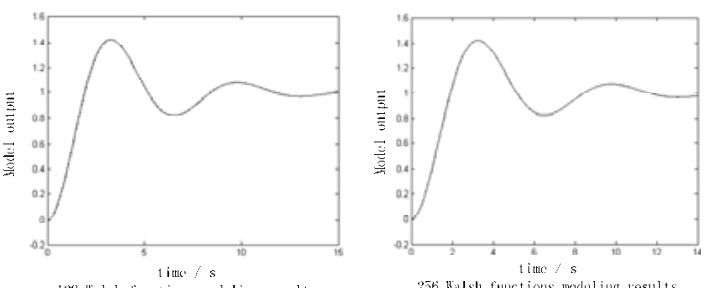

Fig. 2 Modeling results of different number of Walsh function

A comparison between IMU outputs with/without using the pre-filtering Kalman filter was made in order to study how the pre-filtering improves the IMU outputs. In the simulation test, the dynamics of the trajectory are known, i.e. the position, attitude and velocity, and the original IMU outputs could be deduced from this information. After adding noise to the original IMU outputs, the simulated raw IMU outputs corresponding to the flight trajectory could be obtained. Simulation time lasts for 60 sec. The output rate of the IMU is $100 \mathrm{~Hz}$. Figure 3 depicts the IMU outputs that have large errors from the known trajectory.

Table 2. Parameters of flight trajectory

\begin{tabular}{c|c|c|c}
\hline $\begin{array}{c}\text { Initial position } \\
\text { (latitude, longitude, } \\
\text { high) (degree) }\end{array}$ & $45^{\circ}$ & $45^{\circ}$ & 0 \\
\hline $\begin{array}{c}\text { Initial velocity } \\
\text { (east, north, up) (m/s) }\end{array}$ & 1 & 1 & 0 \\
\hline $\begin{array}{c}\text { Initial acceleration } \\
(\mathrm{m} / \mathrm{s} 2)\end{array}$ & 0 & 0 & $45^{\circ}$ \\
\hline $\begin{array}{c}\text { Initial attitude } \\
\text { (roll, pitch, heading) } \\
\text { (degree) }\end{array}$ & 0 & 0 & 00 \\
\hline Simulation time(s) & \multicolumn{3}{|c}{0.01} \\
\hline Simulation time step (s) & \multicolumn{3}{|c}{ Constant acceleration $=0.5 \mathrm{~g}$} \\
\hline
\end{tabular}

After optimally estimating the IMU outputs by the prefiltering Kalman filter, most of the IMU random drift errors were removed, and the filtered IMU outputs are 
shown in Figure 4, indicating an improvement in IMU outputs.

\subsection{Main Kalman Filter Testing Results}

To test the performance of the federated Kalman filter, the comparison of the estimates from these two configurations, i.e. the federated and centralised Kalman filter, is carried out.
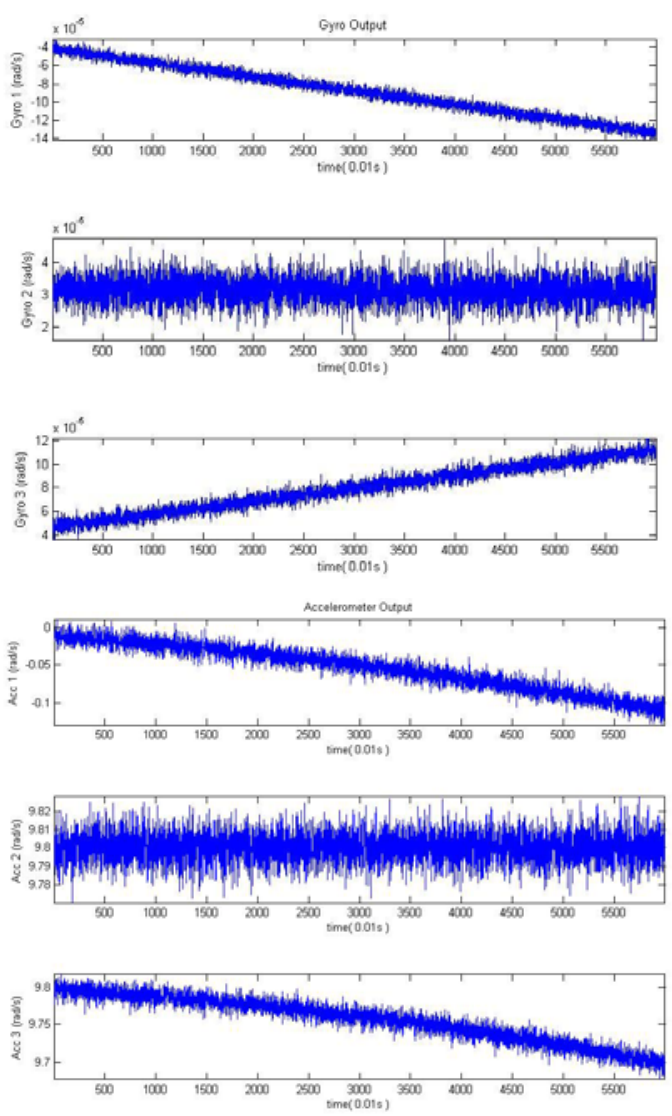

Fig. 3 Original outputs of IMU

In the centralised Kalman filter structure, the IMU random drifts errors are estimated together with other state variables. The optimal navigation solutions of position, attitude and velocity, represented as the latitude, longitude, earth-level velocity and roll, pitch, heading (compensated by the estimated errors derived from the Kalman filter) are shown in Figure 5.

In the federated Kalman filter, as the IMU random drift errors are already estimated during the pre-filtering, the main Kalman filter doesn't need to estimate the IMU errors again. To compare with the results of the centralised Kalman filter, the compensated position, attitude and velocity are given in Figure 6. From Figure 6 it can be observed that the federated Kalman filter delivers the same precision of estimates as the centralised Kalman filter approach, however the computing time is greatly reduced because of the simplified state transition matrix. Hence, the update rate of the estimates can be increased, which is helpful when implementing the Kalman filter for real-time applications.

\subsection{Ultra-Tight GPS/INS Receiver Experimental Results}

As shown in the previous section, the federated Kalman filter structure velocity estimates with the same accuracy as the centralised Kalman filter.
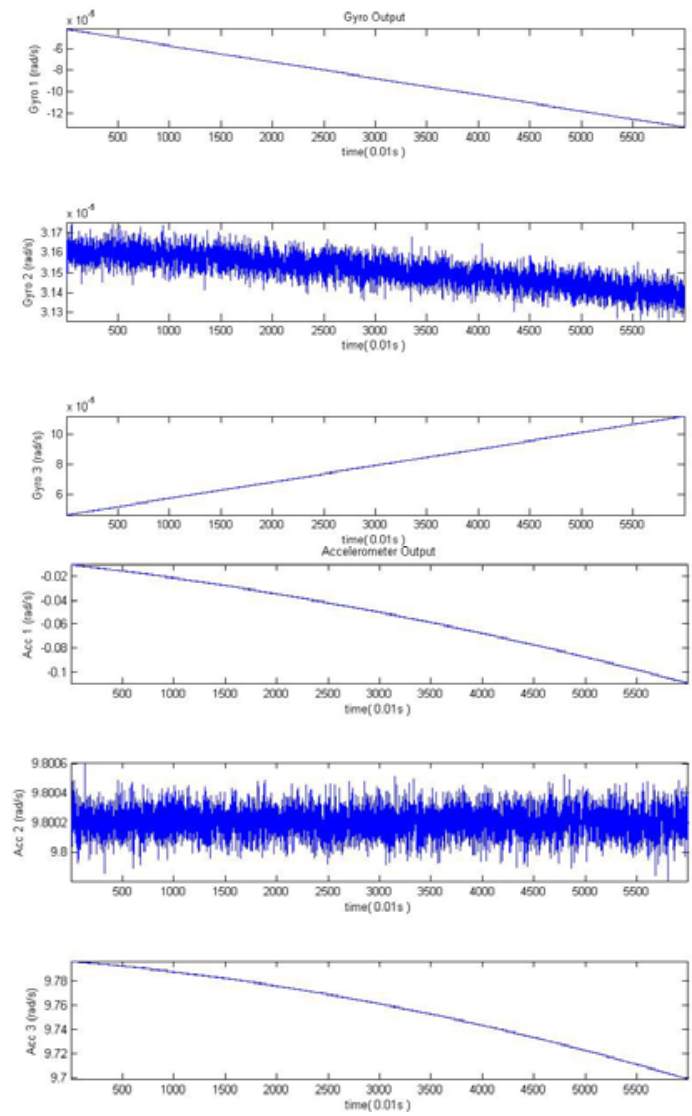

Fig. 4 Filtered outputs of IMU
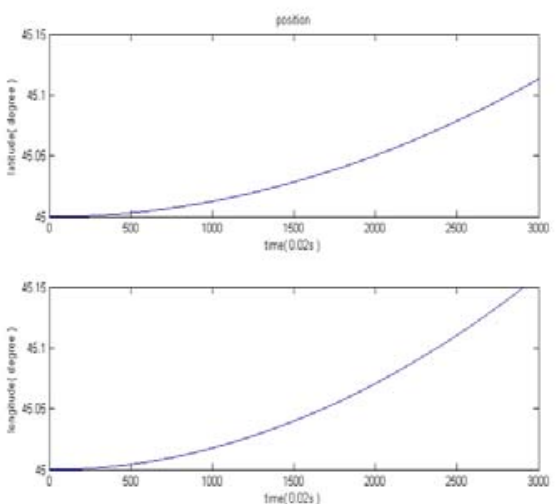

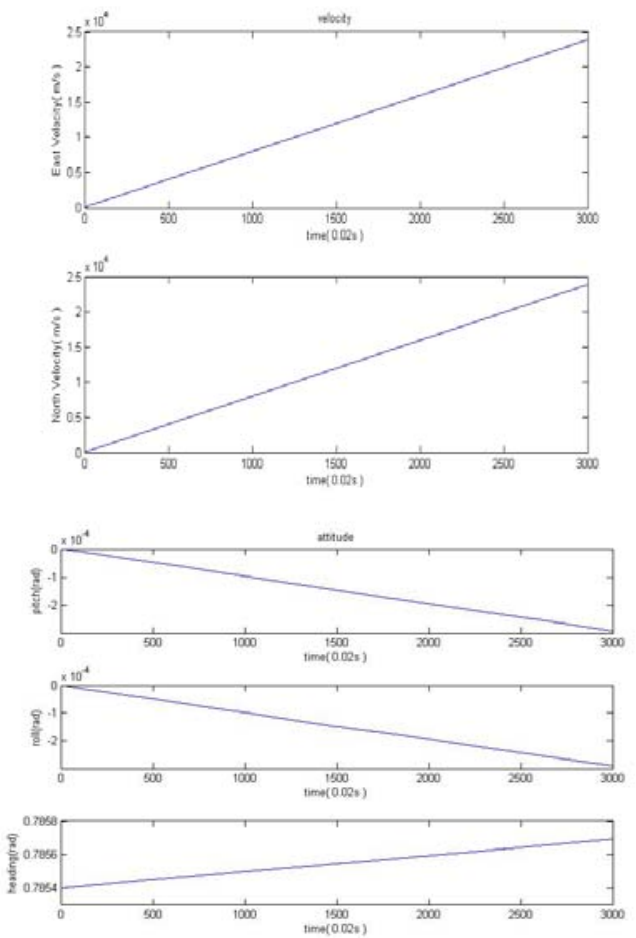

Fig. 5 Navigation solutions based on the centralised Kalman filter structure

Therefore the accuracies of the aiding Doppler for both configurations is of the same level, which means that the ultra-tight GPS/INS receiver based on the federated Kalman receiver has the same quality of output as the centralised Kalman filter, but benefits from faster compution.

Figure 7 illustrates the performance of the ultra-tightly integrated GPS/INS receiver and the stand-alone GPS receiver. The tracking loop bandwidth is set at $12 \mathrm{~Hz}$. Normally, by comparing the power of the carrier tracking loop with the tracking threshold it can be determined if the tracking loop is in FLL or PLL mode, which illustrates the stability and accuracy of the tracking loop. As mentioned above, the FLL means lower measurements accuracy. For better tracking effect, the tracking mode should quickly switch from FLL to PLL. From Figure 7 it can be shown that when the dynamics of the simulated trajectory is high, the stand-alone GPS receiver tracking loop had large tracking errors and remained in the FLL mode. Once the dynamics become larger or the bandwidth is reduced, it will lose lock. However, the ultra-tightly integrated receiver could remain in the PLL tracking mode with the same dynamics, therefore it can be concluded that the ultratight integrated receiver is more robust for the high dynamic applications.

Usually the variety of the code loop power represents the stability of the code tracking loop. If it is large enough to exceed the threshold, the code tracking loop will lose lock. Because the carrier tracking loop relies on the stability of the code tracking loop, it will also lose lock at the same time. Figure 8 shows that the variety of code loop power of the ultra-tight receiver is smaller than that of a stand-alone receiver, meaning it has more stable tracking capability.

The Q measurements represent the tracking loop errors. Figure 9 demonstrates that the ultra-tight receiver has smaller tracking errors than the stand-alone receiver under the same dynamic conditions, i.e. more accurate measurements could be obtained from the tracking loop.
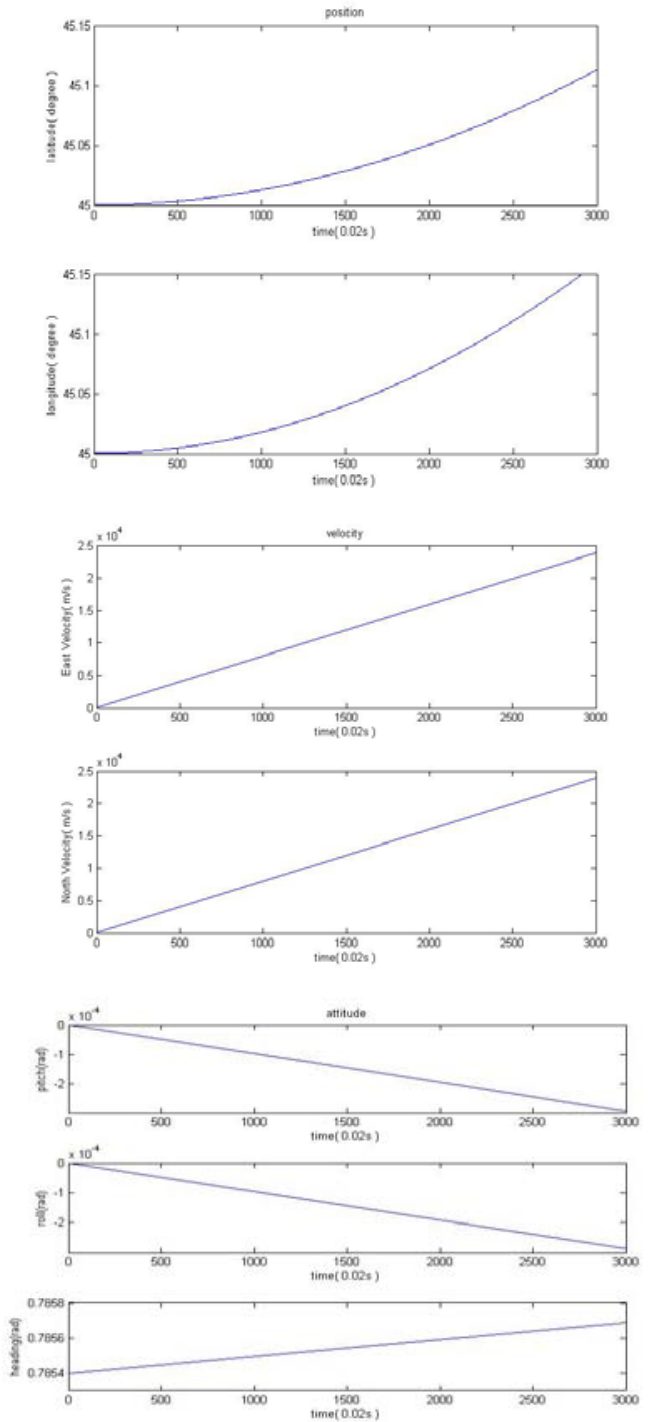

Fig. 6 Navigation solutions based on the federated Kalman filter structure 


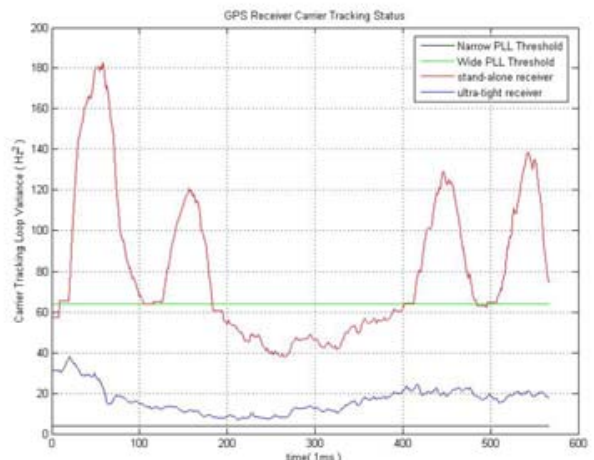

Fig. 7 Comparison of tracking loop status between the ultra tight integration and stand-alone receiver

\section{Concluding Remarks}

In this paper a federated Kalman filter structure for the ultra-tight integration of GPS/INS/PL has been investigated, in which the IMU random errors are estimated and compensated for by a pre-filtering Kalman filter. In this way the computational burden can be separated into different parts and processed in parallel. The pivotal technique to implement this structure, the dynamic modelling method of the IMU based on the Walsh function and its transform, is investigated and the simulation experiments have demonstrated its accuracy and feasibility. The Kalman filter was implemented on the IMU dynamic model. Test results have shown that the IMU errors can be effectively removed by pre-filtering so that the accuracy of the aiding Doppler derived from the integrated Kalman filter is the same as that of the centralised Kalman filter, but requires less computation time. This federated Kalman filter structure is therefore especially attractive, especially for real-time applications.

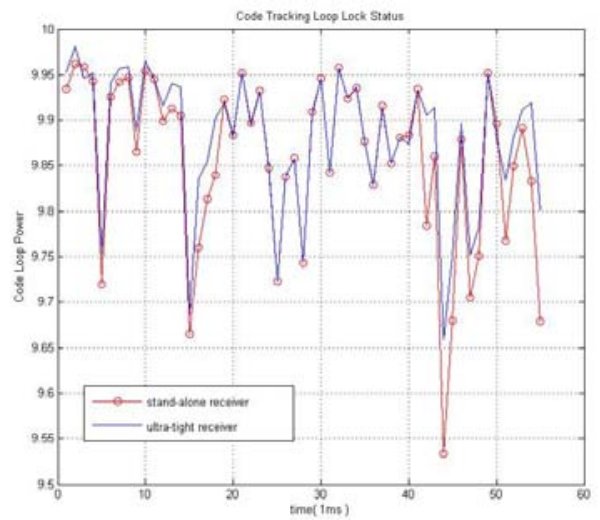

Fig. 8 Code tracking loop status

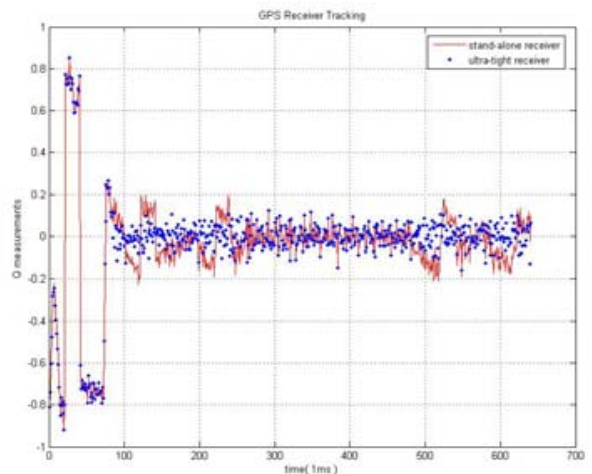

Fig. 9 Tracking loop Q measurements

\section{Acknowledgments}

This research is supported by an ARC (Australian Research Council) - Discovery Research Project on 'Robust Positioning Based on Ultra-tight Integration of GPS, Pseudolites and Inertial Sensors'. The authors would like to thank Prof. Chris Rizos for his valuable comments on the early version of this paper.

\section{References}

Li D., Sun Y. (2004) Research of The Dynamic Modelling of FOG Based on Walsh Function. Ship Engineering, Dec, 2004

Alban S., Akos D., Rock S. \& Gebre-Egziobher D. (2003) Performance Analysis and Architectures for INS-Aided GPS tracking loops. Institute of Navigation - NTM, Anaheim, CA, 22-24 January, 611-622.

Kaplan E.D. (1996) Understanding GPS: Principles and Applications. Artech House, MA. 119-207

Tsui J.B.Y. (2000) Fundamentals of Global Positioning Receivers - A Software Approach. John Wiley \& Sons, Inc. $165-190$

Beser J., Alexander S., Crane R., Rounds S., Wyman J., Baeder B. (2002) TrunavTM: A Low-Cost Guidance/Navigation Unit Integrating a SAASM-based GPS and MEMS IMU in a Deeply Coupled Mechanization. 15th Int. Tech. Meeting of the Satellite Division of the U.S. Inst. of Navigation, Portland, OR, 24-27 September, 545-555.

Babu R., Wang J. (2004) Comparative study of interpolation techniques for ultra-tight integration of GPS/INS/PL sensors. GNSS 2004, Sydney, Australia, 6-8 Dec.

Babu R., Wang J. (2004) Improving the Quality of IMUDerived Doppler Estimates for Ultra-Tight GPS/INS Integration. GNSS 2004, Rotterdam, The Netherlands, 1619 May.

Farrell J.A. \& Barth M. (1999) The Global Positioning System and Inertial Navigation. McGraw-Hill. 
Hentschel T. \& Fettweis G. (2000) Sample Rate Conversion for Software Radio. IEEE Communications Magazine, August, 2-10.

Mitra S.K. (1999) Digital Signal Processing - A Computer Based Approach. Tata McGraw-Hill Edition, India, ISBN 0-07-463723-1.

Vaidyanathan P.P. (1990) Multirate Digital Filter, Filter Banks, Polyphase Networks, and Applications: A Tutorial. Proc. IEEE, 78, 56-93.

Wang J., Dai L., Tsuiji T., Rizos C., Grejner-Brzezinska D., Toth C. (2001) GPS/INS/Pseudolite Integration:
Concepts, Simulation and Testing. 14th Int. Tech. Meeting of the Satellite Division of the U.S. Inst. of Navigation, Salt Lake City, Ohio, 11-14 September, 27082715 .

Ward P. (1998) Performance Comparisons Between FLL, PLL and a Novel FLL-Assisted PLL Carrier Tracking Loop Under RF Interference Conditions. 11th Int. Tech. Meeting of the Satellite Division of the U.S. Inst. of Navigation, Nashville, Tennessee, 15-18 September, 783795. 